\title{
ARTICLE Scaling behavior of nanoimprint and nanoprinting lithography for producing nanostructures of molybdenum disulfide
}

\author{
Mikai Chen, Hossein Rokni, Wei Lu and Xiaogan Liang
}

Top-down lithography techniques are needed for manufacturing uniform device structures based on emerging 2D-layered materials. Mechanical exfoliation approaches based on nanoimprint and nanoprint principles are capable of producing ordered arrays of multilayer transition metal dichalcogenide microstructures with a high uniformity of feature dimensions. In this study, we present a study on the applicability of nanoimprint-assisted shear exfoliation for generating ultrathin monolayer and few-layer $\mathrm{MoS}_{2}$ structures as well as the critical limits of feature dimensions produced via such nanoimprint and nanoprint-based processes. In particular, this work shows that give a lateral feature size of $\mathrm{MoS}_{2}$ structures that are pre-patterned on a bulk stamp, there exists a critical thickness or aspect ratio value, below which the exfoliated layered structures exhibit major defects. To exfoliate a highquality, uniform monolayer or few-layer structures, the characteristic lateral feature sizes of such structures need to be in the sub-100 nm regimes. In addition, the exfoliated $\mathrm{MoS}_{2}$ flakes of critical thicknesses exhibit prominent interlayer twisting features on their cleaved surfaces. Field-effect transistors made from these $\mathrm{MoS}_{2}$ flakes exhibit multiple (or quasi-analog-tunable) charge memory states. This work advances the knowledge regarding the limitations and application scope of nanoimprint and nanoprint processes in manufacturing nano/microstructures based on layered materials and provides a method for producing multi-bit charge memory devices.

Keywords: 2D materials; charge trapping; memory; $\mathrm{MoS}_{2}$; nanoimprint; nanoprint

Microsystems \& Nanoengineering (2017) 3, 17053; doi:10.1038/micronano.2017.53; Published online: 11 September 2017

\section{INTRODUCTION}

Two dimensional (2D)-layered transition metal dichalcogenides (TMDCs) (for example, $\mathrm{MoS}_{2}, \mathrm{WSe}_{2}$, and $\mathrm{WS}_{2}$ ) have been widely studied as attractive material candidates for making electronic, electrical, photonic, biological, and chemical sensing devices with either significantly improved performance or a new functionality ${ }^{1-9}$. Despite the breakthroughs and progress in demonstrating TMDC-based prototype devices, the research and industry communities still lack scalable manufacturing techniques that are capable of producing orderly arranged, high-quality, highly uniform TMDC device structures over wafer-scale areas. In particular, the community needs top-down lithography manner techniques that are capable of producing pre-structured TMDC nano/microstructures with deterministic and uniform feature dimensions and physical properties. Our research team recently developed and investigated a nanoimprint/nanoprint-based method that is capable of producing multilayer $\mathrm{MoS}_{2}$ device structure arrays with a high device-todevice consistency in feature dimensions and electronic/biosensing characteristics $^{10}$. This approach is called nanoimprint-assisted shear exfoliation (NASE). In our previous works, NASE processes were demonstrated to be capable of generating multilayer TMDC structures with characteristic lateral feature sizes in the range of 7 to $25 \mu \mathrm{m}$ and vertical thicknesses in the range of $20-200 \mathrm{~nm}^{10,11}$. Additional effort is needed to identify the limits of the critical feature dimensions produced by NASE processes and further extend the application scope of NASE for producing ultrathin monolayer and few-layer TMDC device structures.

In this study, we present a study on the important limits of the feature dimensions of $\mathrm{MoS}_{2}$ nanostructures (the most widely studied TMDC structures) produced by shear exfoliation in combination with transfer printing. In particular, we experimentally evaluated the viability of nanoimprint and nanoprint processes for producing ultrathin monolayer and few-layer $\mathrm{MoS}_{2}$ structures. More specifically, our work shows that the quality of mechanically exfoliated $\mathrm{MoS}_{2}$ nano/microstructures highly depends on the aspect ratio (or the ratio between feature thickness (or height) and lateral feature size) of pre-patterned structures on a bulk $\mathrm{MoS}_{2}$ stamp. For exfoliated $\mathrm{MoS}_{2}$ features with a given lithographically defined characteristic lateral size, the vertical thicknesses (or aspect ratios) of these features have a critical limit, below which the exfoliated $\mathrm{MoS}_{2}$ features exhibit major defects that are caused by mechanical exfoliation processes. For example, for exfoliated $\mathrm{MoS}_{2}$ features with lateral feature sizes larger than $5 \mu \mathrm{m}$, the critical aspect ratio is estimated to be approximately 1:430; for exfoliated $\mathrm{MoS}_{2}$ nanostructures with lateral sizes smaller than $1 \mu \mathrm{m}$, the critical aspect ratio is approximately $1: 47$. This strongly implies that to generate highquality ultrathin monolayer or few-layer $\mathrm{MoS}_{2}$ structures using nanoimprint and nanoprint approaches, the lateral dimension sizes of pre-patterned $\mathrm{MoS}_{2}$ features on a bulk stamp need to be set in the sub-100 nm regime. In addition, the exfoliated $\mathrm{MoS}_{2}$ structures, whose thicknesses or aspect ratios are close to corresponding critical values, exhibit prominent top-view Moiré patterns on the cleaved surfaces, indicating the existence of interlayer twisting features in the $\mathrm{MoS}_{2}$ layers close to these cleaved surfaces. Fieldeffect transistors made from such $\mathrm{MoS}_{2}$ structures exhibit multiple (or quasi-analog-tunable) charge memory states, which could be further exploited to make analog computing and multi-bit memory devices. 


\section{MATERIALS AND METHODS}

Figure 1 schematically illustrates the nanofabrication method under study, which involves a NASE process followed by a transfer-printing (TP) step and is therefore referred to as the NASE +TP method. Specifically, this NASE+TP method includes (a) prefabrication of a bulk $\mathrm{MoS}_{2}$ stamp-bearing protrusive to-beexfoliated device structures with deterministic lateral sizes and thicknesses, (b) room temperature nanoimprinting of protrusive to-be-exfoliated $\mathrm{MoS}_{2}$ features into a polydimethylsiloxane (PDMS) stamp, (c) shear exfoliation of imprinted $\mathrm{MoS}_{2}$ features into the PDMS stamp using a roller tool, (d) elastic recovery of the PDMS stamp, elevating the as-exfoliated $\mathrm{MoS}_{2}$ structures onto the flat PDMS surface, and (e) transfer printing of the $\mathrm{MoS}_{2}$ features onto the target substrate via a thermal release process. In this work, the bulk $\mathrm{MoS}_{2}$ ingots for making nanoimprint stamps are from SPI (SPI Supplies, West Chester, PA, USA) (sample size of $\sim 1 \mathrm{~cm}^{2}$ ). The prepatterning of the $\mathrm{MoS}_{2}$ stamps with protrusive microstructures (that is, the structures with microscale characteristic lateral feature sizes) was performed using a method previously reported by our group $^{12}$. To pre-pattern nanostructures with various nanoscale lateral feature sizes on the stamps, we exploited a series of different lithography techniques, including block copolymer $(\mathrm{BCP})$ self-assembly ${ }^{13}$, nanoimprint lithography $(\mathrm{NIL})^{12}$, and nanoparticle (NP) lithography. Specifically, polystyrene-blockpolydimethylsiloxane (PS-b-PDMS) copolymers were used for generating sub-10 nm size line-spacing patterns. The details of the $B C P$ processes can be found in our previously published works ${ }^{13-15}$. NIL processes were performed to generate polymeric nanopatterns with characteristic lateral feature sizes in the range of $100 \mathrm{~nm}$ to $1 \mu \mathrm{m}^{12}$. Furthermore, $80 \mathrm{~nm}$ Au NPs were randomly dispersed onto $\mathrm{MoS}_{2}$ ingot surfaces to form NP cluster patterns with characteristic lateral sizes ranging from 80 to $500 \mathrm{~nm}$, which can serve as etching masks for generating protrusive $\mathrm{MoS}_{2}$ mesas with various lateral sizes (note that such randomly distributed NP/ cluster patterns are not used to create any ordered device structures but purposely generate $\mathrm{MoS}_{2}$ mesas with a broad range of lateral feature sizes and therefore enable the study of the effects of different aspect ratios of to-be-exfoliated $\mathrm{MoS}_{2}$ nanostructures on the exfoliation results). With such BCPs, NIL-generated polymeric and NP/cluster nanopatterns as the etching masks, protrusive tobe-exfoliated nanostructures on the bulk $\mathrm{MoS}_{2}$ stamps were formed using $\mathrm{SF}_{6}$ reactive ion etching (RIE) (processing parameters: $\mathrm{SF}_{6}$, flow rate: $20 \mathrm{sccm}$, chamber pressure: $20 \mathrm{mTorr}, \mathrm{RF}$ power: $200 \mathrm{~W}$ ). The etching rate was $\sim 100 \mathrm{~nm} \mathrm{~min}{ }^{-1}$. Figure 2 displays the scanning electron micrographs (SEMs) of several representative $\mathrm{MoS}_{2}$ stamps bearing the protrusive nano/microstructures with various characteristic lateral feature sizes and vertical heights (or thicknesses), which include (a) 8-nm half-pitch line-spacing structures patterned from PS-b-PDMS BCP features, (b) 100-nm half-pitch line-spacing structures formed by NIL processes, (c) $80-\mathrm{nm}$ and (d) hundreds of $\mathrm{nm}$-sized protrusive mesas patterned from randomly distributed Au NPs and clusters, and (e) $10-\mu \mathrm{m}$ protrusive mesas formed using regular photolithography followed by RIE. In summary, the fabricated $\mathrm{MoS}_{2}$ stamps bear to-be-exfoliated nano/microstructures with characteristic lateral feature sizes ranging from $8 \mathrm{~nm}$ to $50 \mu \mathrm{m}$ and vertical feature heights (or thicknesses) ranging from $0.8 \mathrm{~nm}$ (monolayer structures) to $500 \mathrm{~nm}$ ( 770 layers).

During the NASE+TP process, a lab-made motorized roller tool is used to firmly press the $\mathrm{MoS}_{2}$ stamp against the PDMS film (vertical gauge pressure of $\sim 2 \times 10^{6} \mathrm{~Pa}$ ) and simultaneously generate a shear displacement (relative velocity $0.1-1 \mathrm{~mm} \mathrm{~s}^{-1}$ ) between the protrusive $\mathrm{MoS}_{2}$ structures mechanically imprinted into PDMS and the bulk stamp via the friction force between the rotating roller and the bulk stamp ${ }^{10,11}$. Because of this shear displacement, the imprinted $\mathrm{MoS}_{2}$ layers can be exfoliated away from the stamp along the shear direction ${ }^{10,11}$. After this shear exfoliation step, the vertical imprint pressure is released, and the surface of the elastic PDMS film quickly restores back to the flat morphology, forming a flat PDMS stamp that bears exfoliated $\mathrm{MoS}_{2}$ nano- and microstructures. During the NASE process, the thicknesses of the exfoliated $\mathrm{MoS}_{2}$ flakes are mainly determined by the imprinting depth (that is, the pre-defined height) of the protrusive $\mathrm{MoS}_{2}$ structures that are pre-patterned on the bulk $\mathrm{MoS}_{2}$ stamp ${ }^{10,11}$. During the transfer-printing step, the $\mathrm{MoS}_{2}$ nanoand microstructures on the PDMS stamp are mechanically pressed against the target Si substrate that is coated with 300-nm-thick thermally grown $\mathrm{SiO}_{2}$. Afterwards, the PDMS stamp is heated up to $200{ }^{\circ} \mathrm{C}$ using a heater, and the $\mathrm{MoS}_{2}$ nano- and microstructures are thermally released from the PDMS stamp and subsequently transferred onto the target $\mathrm{Si} / \mathrm{SiO}_{2}$ substrate.

Scanning electron microscopy (SEM) images of as-exfoliated $\mathrm{MoS}_{2}$ nano- and microstructures were taken using an in-line field-emission SEM (Manufacturer: Hitachi High Technologies America, Schaumburg, IL, USA; Model Number: Hitachi SU8000 In-line FE-SEM) with an accelerating voltage of $2 \mathrm{kV}$. High-resolution transmission electron microscopy (HRTEM) images of the cleaved surfaces of the $\mathrm{MoS}_{2}$ structures were obtained using a JEOL $2010 \mathrm{~F}$ analytical electron microscope with an accelerating voltage of $200 \mathrm{kV}$.

\section{RESULTS}

Figure 3 displays the SEM images of the representative $\mathrm{MoS}_{2}$ nano/microstructures produced by the NASE+TP processes
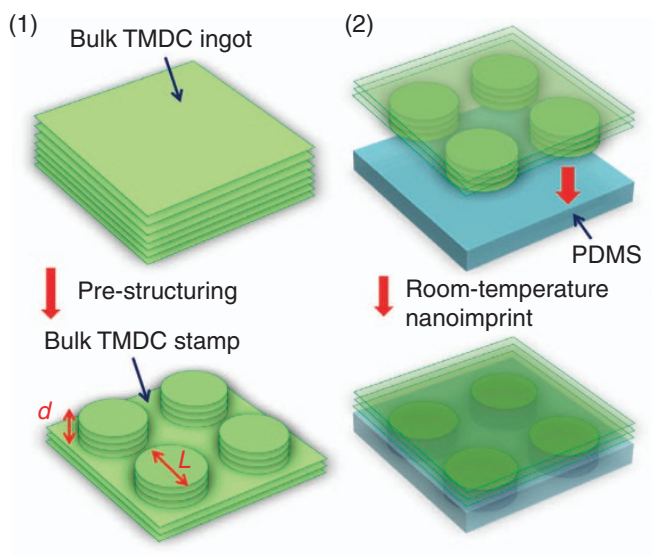

(4)

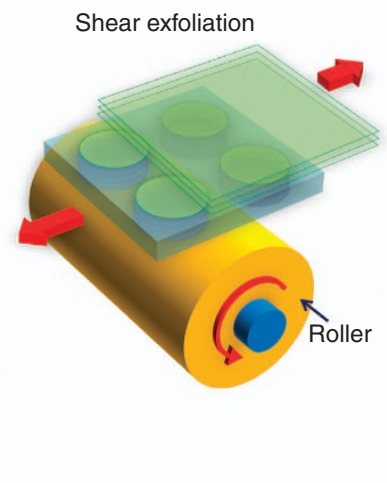

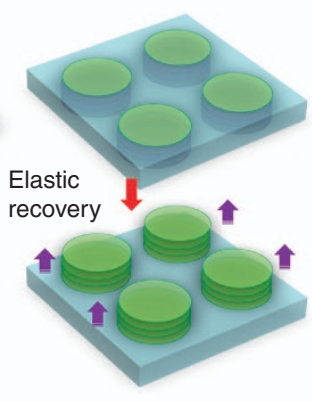

(5)

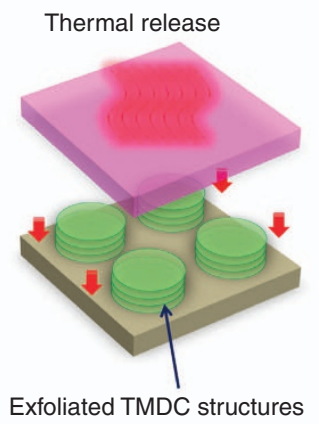

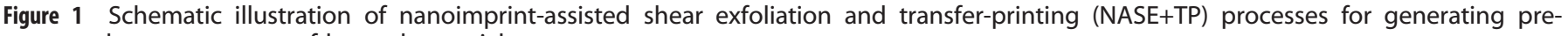
patterned nanostructures of layered materials. 


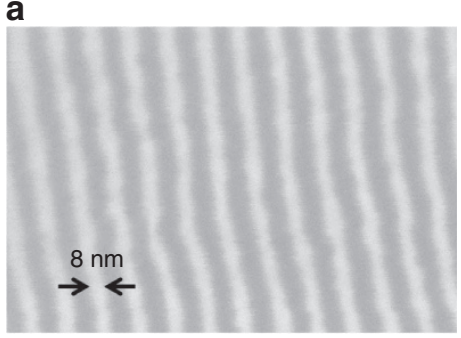

b

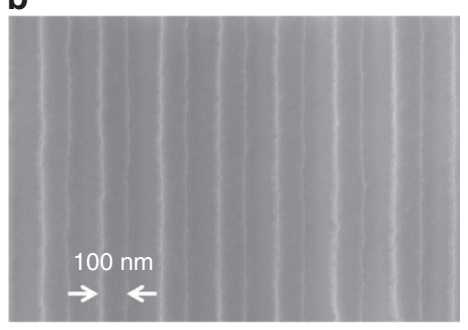

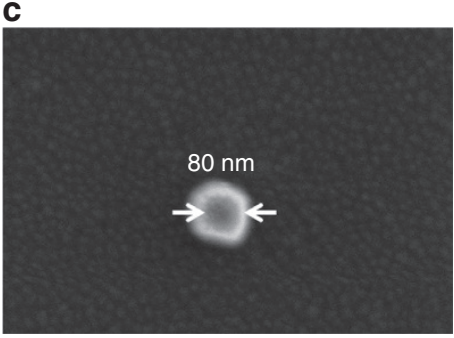

d

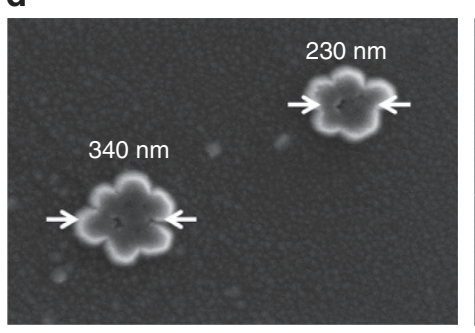

e

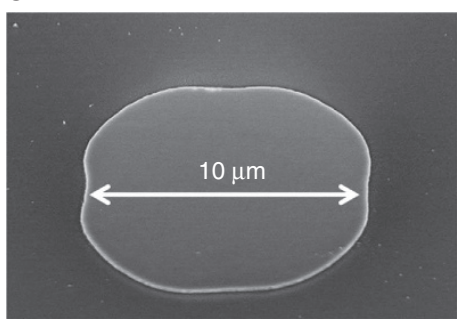

Figure 2 SEM images of a set of representative bulk $\mathrm{MoS}_{2}$ stamps bearing (a) 8-nm half-pitch line-spacing patterns, (b) 100-nm half-pitch linespacing patterns, (c) 80-nm pillars, (d) hundreds of nm-sized mesas, and (e) $10-\mu \mathrm{m}$ mesas.

performed under the same processing condition (vertical gauge pressure of $\sim 2 \times 10^{6} \mathrm{~Pa}$, shear velocity of $\sim 0.5 \mathrm{~mm} \mathrm{~s}^{-1}$ ), which have relatively large feature aspect ratios (that is, feature thickness $(d)$ /characteristic lateral feature size $(L))$ and exhibit a high feature quality. These nano/microstructures include (a) an 8-nm half-pitch, 1.3-nm-thick line-spacing structures (aspect ratio of approximately 1:6), (b) a 100-nm half-pitch, 3.3-nm-thick line-spacing structures (aspect ratio of $\sim 1: 30$ ), (c) 80-150-nm size, 10-nm-thick mesas (aspect ratios: 1:8-15), (d) 5- $\mu \mathrm{m}$ size, 25-nm-thick flakes (aspect ratio of 1:200), (e) 20- $\mu \mathrm{m}$ size, 40-nm-thick flakes (aspect ratio of 1:500), and (f) a 380-nm size, 20-nm-thick flake (aspect ratio of approximately $1: 20$ ). To further verify that the produced nano- and microstructures are made of $\mathrm{MoS}_{2}$, we used the energy dispersive $X$-ray spectroscopy (EDS) module in the SEM system (Model: Tescan MIRA3 FEG SEM; acceleration voltage: $5 \mathrm{kV}$; dwell time: $200 \mu \mathrm{s})$ to acquire EDS spectra and corresponding elemental mapping images from the regions bearing the target nano- and microstructures. For example, Figure $3 \mathrm{~g}$ displays the integral EDS spectrum obtained from the nanoscale flake shown in Figure $3 f$. This EDS spectrum clearly shows the peak associated with $\mathrm{MoS}_{2}$. In addition, Figure $3 \mathrm{~h}$ shows the corresponding EDS elemental mapping image of Mo (Red) and Si (Green) in which the $\mathrm{MoS}_{2}$ feature exhibits an identifiable contrast against the $\mathrm{Si}$ background. This demonstrates that EDS can be used as a tool for identifying sub-micrometer $\mathrm{MoS}_{2}$ features. These results also indicate that the observed nano- and microstructures are indeed made of $\mathrm{MoS}_{2}$.

Figure 4 displays the SEM images of the representative NASE+TP-produced $\mathrm{MoS}_{2}$ nano- and microstructures with relatively small aspect ratios, which exhibit major defects caused by shear exfoliation. These exfoliated $\mathrm{MoS}_{2}$ nano- and microstructures include (a) an $8 \mathrm{~nm}$ half-pitch, $0.6 \mathrm{~nm}$-thick line-spacing structures (aspect ratio approximately $1: 14$ ), (b) a $100 \mathrm{~nm}$ halfpitch, $2 \mathrm{~nm}$-thick line-spacing structures (aspect ratio approximately 1:50), (c) hundreds of nanometer-sized, $3.5 \mathrm{~nm}$-thick mesas (aspect ratio approximately 1:29), (d) $5 \mu \mathrm{m}$ size, $8 \mathrm{~nm}$-thick flakes (aspect ratio approximately 1:625), (e) $10 \mu \mathrm{m}$ size, $20 \mathrm{~nm}$-thick lines (aspect ratio approximately 1:500), (f) $20 \mu \mathrm{m}$ size, $33 \mathrm{~nm}$-thick flakes (aspect ratio approximately 1:600), and (g) $50 \mu \mathrm{m}$ wide, $70 \mathrm{~nm}$-thick (average thickness) film (aspect ratio approximately $1: 710)$.

\section{DISCUSSION}

Our NASE+TP test results of the $\mathrm{MoS}_{2}$ nano- and microstructures with various lateral feature sizes and vertical thicknesses indicate that for a given characteristic lateral size of pre-patterned $\mathrm{MoS}_{2}$ a nano- or microstructure on the bulk $\mathrm{MoS}_{2}$ stamp, the vertical thickness (or aspect ratio) of these structures has a critical limit $\left(d_{c}\right)$; below this critical limit to-be-exfoliated $\mathrm{MoS}_{2}$ nano- and microstructures have a poor mechanical rigidity, and as-exfoliated nano/structures typically exhibit major defective features (that is, a very low yield of high-quality $\mathrm{MoS}_{2}$ structures that are free of mechanical damage) that are caused by the shear exfoliation. More specifically, Figure 5 plots the experimentally determined critical thickness $\left(d_{c}\right)$ values as a function of characteristic lateral feature sizes $(L)$. As shown in Figure 5, for the exfoliated $\mathrm{MoS}_{2}$ structures with lateral feature sizes larger than $5 \mu \mathrm{m}$, the critical aspect ratio is estimated to be 1:430 \pm 20 , whereas for the $\mathrm{MoS}_{2}$ nanostructures with lateral sizes smaller than $1 \mu \mathrm{m}$, the critical aspect ratio to assure a damage-free exfoliation is approximately $1: 47 \pm 30$. This result implies that to produce high-quality ultrathin monolayer or few-layer $\mathrm{MoS}_{2}$ structures using NASE+TP or other mechanical exfoliation processes, the lateral dimensions of the protrusive to-be-exfoliated $\mathrm{MoS}_{2}$ features on the bulk stamp need to be patterned in sub-100-nm regimes. The critical feature aspect ratio required for generating high-quality $\mathrm{MoS}_{2}$ structures increases with the reduction of the targeted feature thickness (or the targeted lateral size). Thus, in comparison with the production of relatively thick multilayer $\mathrm{MoS}_{2}$ structures (typically thicker than $10 \mathrm{~nm}$ ), the production of ultrathin monolayer or fewlayer $\mathrm{MoS}_{2}$ structures using NASE+TP processes needs a larger feature aspect ratio to assure that the $\mathrm{MoS}_{2}$ nanostructures have a sufficiently high rigidity and can survive shear exfoliation processes. This trend of requiring larger feature aspect ratios for high-yield exfoliation of monolayer/few-layer structures probably occurs because when the thickness of to-be-exfoliated $\mathrm{MoS}_{2}$ structures is pre-defined in monolayer/few-layer regimes (that is, typically, $0.5-7 \mathrm{~nm}$ ), the nanoscale surface roughness of the $\mathrm{MoS}_{2}$ stamp and naturally formed crystal terraces with nanoscale step heights could mechanically interact with as-exfoliated $\mathrm{MoS}_{2}$ nanostructures. Because of the comparable dimension sizes between roughness features and exfoliated ultrathin $\mathrm{MoS}_{2}$ 

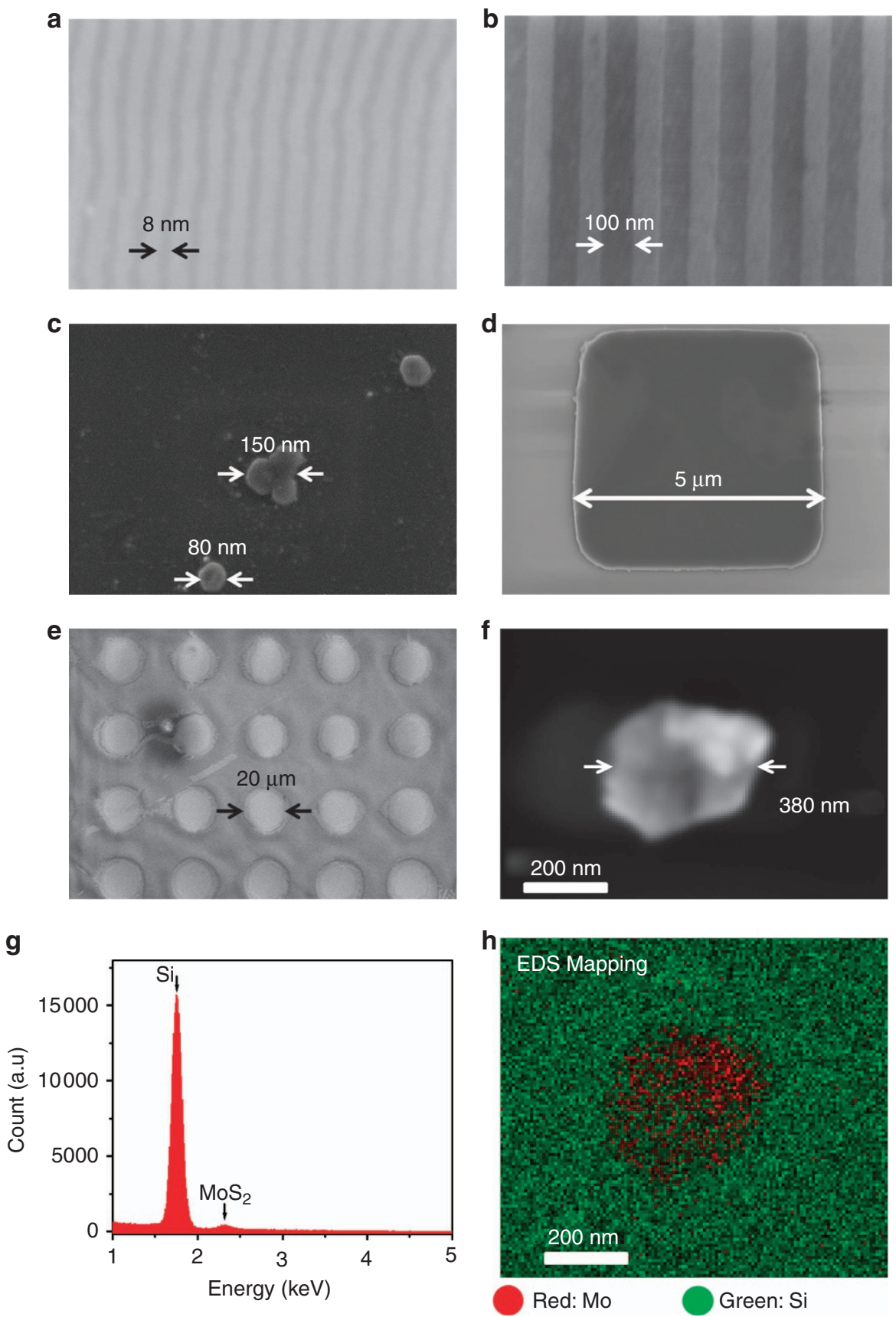

Figure 3 SEM images of representative good-quality $\mathrm{MoS}_{2}$ nano- and microstructures produced via NASE+TP processes, including (a) 8 nm half-pitch, $1.3 \mathrm{~nm}$-thick line-spacing patterns, (b) $100 \mathrm{~nm}$ half-pitch, $3.3 \mathrm{~nm}$-thick line-spacing patterns, (c) $80-150 \mathrm{~nm}$ size, $10 \mathrm{~nm}$-thick mesas, (d) $5 \mu \mathrm{m}$ size, $25 \mathrm{~nm}$-thick flakes, (e) $20 \mu \mathrm{m}$ size, $40 \mathrm{~nm}$-thick flakes, and (f) a $380 \mathrm{~nm}$ size, $20 \mathrm{~nm}$-thick flake. (g) The EDS spectrum acquired from the $\mathrm{MoS}_{2}$ nanostructure shown in (f). (h) The EDS elemental mapping of Mo and Si.

structures, such mechanical interaction easily leads to severe damage to the as-exfoliated $\mathrm{MoS}_{2}$ structures. Therefore, the thinner to-be-exfoliated structure typically needs a larger feature aspect ratio (that is, a higher structure rigidity) to survive in the exfoliation process. For the exfoliation of relatively thick multilayer structures (typically thicker than $7 \mathrm{~nm}$ ), such an effect on the surface roughness features is not expected to be significant because of the large discrepancy between exfoliated $\mathrm{MoS}_{2}$ structures and surface roughness features regarding their dimension sizes. The most important physical significance of the critical thickness (or aspect ratio) data plotted in Figure 5 is that they provide a quantitative limit profile for the $\mathrm{MoS}_{2}$ feature dimensions enabled by NASE+TP, which is designed to generate $\mathrm{MoS}_{2}$ device structures with a high uniformity in feature size. Although the specific values of critical thicknesses or aspect ratios are expected to be different for various layered materials or various exfoliation methods, the basic scaling behaviors (or trends) of the critical aspect ratios for these different cases are still expected to be similar. This is because the aspect ratios of to-be-exfoliated layered nano- and microstructures always play a critical role in determining the rigidity of such features, which always significantly affects the yield of good-quality features generated by 
a
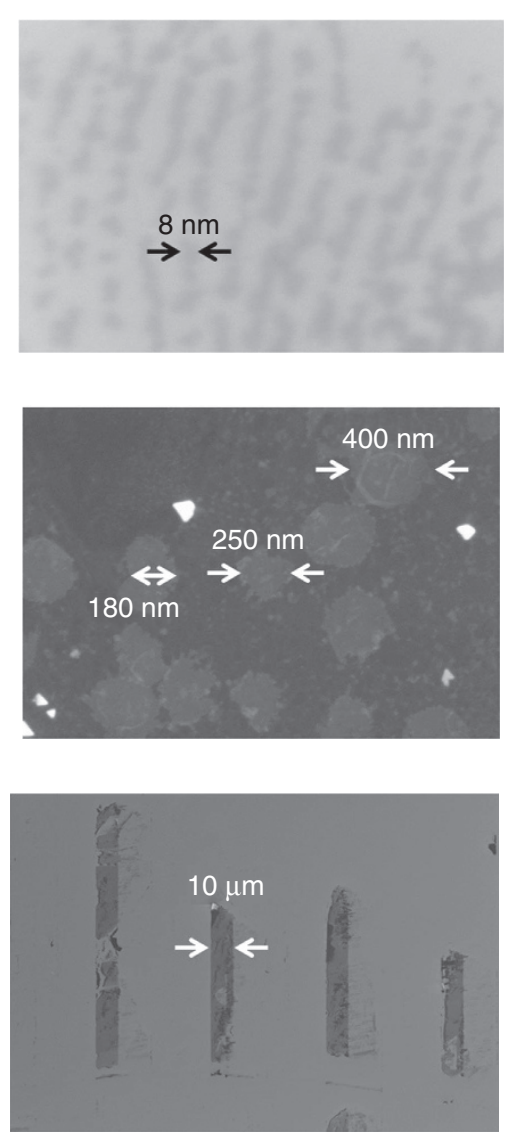

b

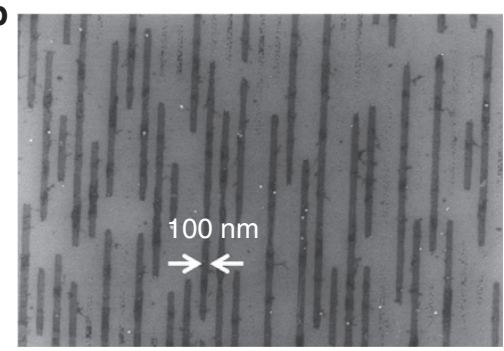

d

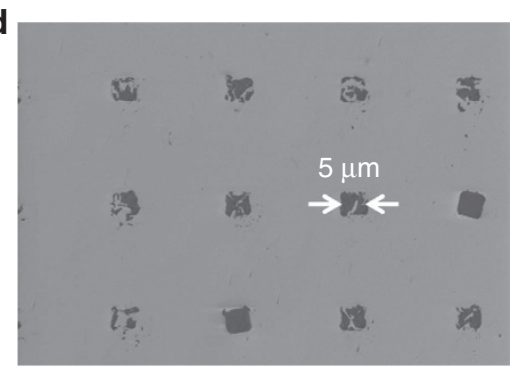

f

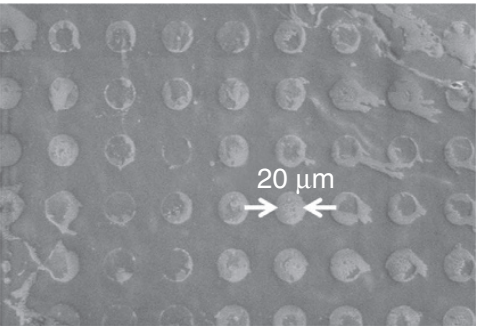

g

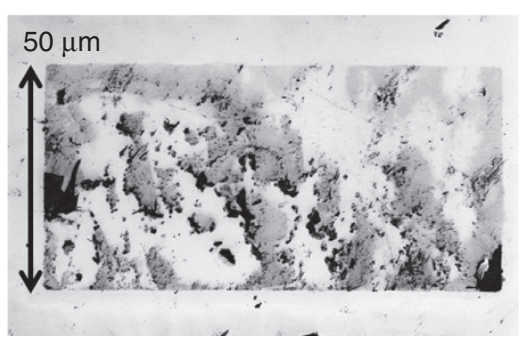

Figure 4 SEM images of representative bad quality $\mathrm{MoS}_{2}$ nano- and microstructures with feature aspect ratios (feature thickness/lateral size ratios) smaller than the critical aspect ratios, which results in major defects in the exfoliated structures: (a) $8 \mathrm{~nm}$ half-pitch, $0.6 \mathrm{~nm}$-thick linespacing patterns, (b) $100 \mathrm{~nm}$ half-pitch, $2 \mathrm{~nm}$-thick line-spacing patterns, (c) hundreds of nm size, $3.5 \mathrm{~nm}$-thick flakes, (d) $5 \mu \mathrm{m}$ size, $8 \mathrm{~nm}$-thick flakes, (e) $10 \mu \mathrm{m}$ size, $20 \mathrm{~nm}$-thick lines, (f) $20 \mu \mathrm{m}$ size, $33 \mathrm{~nm}$-thick flakes, (g) $50 \mu \mathrm{m}$ wide, $70 \mathrm{~nm}$-thick (average thickness) film.

various mechanical exfoliation methods. Furthermore, the method presented here for measuring the critical aspect ratios of NASE +TP-produced $\mathrm{MoS}_{2}$ structures can serve as a generic methodology for determining the critical aspect ratios of various layered materials generated via different exfoliation methods.

Another concomitant effect of the surface roughness and crystal terrace features is that when the aspect ratio (or thickness) of pre-patterned to-be-exfoliated $\mathrm{MoS}_{2}$ structures is close to or smaller than the corresponding critical value, the selectivity for preferred exfoliation of pre-patterned (or targeted) $\mathrm{MoS}_{2}$ layers over undesired exfoliation of unpatterned (or untargeted) $\mathrm{MoS}_{2}$ layers becomes significantly lower. For example, Figure 6 shows an optical micrograph of an array of as-exfoliated 15- $\mu \mathrm{m}$ size, 20-nmthick $\mathrm{MoS}_{2}$ mesas (corresponding critical thickness, $d_{c^{\prime}} \sim 30 \mathrm{~nm}$ ) and the untargeted underlying $\mathrm{MoS}_{2}$ layers. Such untargeted layers are not lithographically patterned but are usually exfoliated due to the poor exfoliation selectivity between pre-patterned and unpatterned $\mathrm{MoS}_{2}$ layers. More specifically, in the case of exfoliation of relatively thick pre-patterned $\mathrm{MoS}_{2}$ structures $\left(d_{\text {» }} d_{\mathrm{c}}\right)$, the shear stress is dominantly accumulated in the pre-patterned $\mathrm{MoS}_{2}$ layers with relatively thick protrusive edges. However, in the case of the exfoliation of relatively thin structures $\left(d<d_{c}\right)$, since the surface roughness is comparable to the pre-patterned feature thickness (or height), a significant portion of the total shear force is exerted on the roughness or crystal terrace features in unpatterned $\mathrm{MoS}_{2}$ layers, resulting in a more even distribution of the shear stress between pre-patterned and unpatterned layers (or regions) and leading to a poor exfoliation selectivity.

We also obtained high-resolution transmission electron micrographs (HRTEMs) of the as-exfoliated $\mathrm{MoS}_{2}$ structures with feature thicknesses $(d)$ close to the corresponding critical values $\left(d_{c}\right)$ and for those with $d_{\wedge} d_{c}$. The exfoliated $\operatorname{MoS}_{2}$ structures with $d \sim d_{c}$ have exfoliation-induced interlayer deformation features. For example, Figures $7 a$ and $b$ show the HRTEM images of the cleaved surfaces of one as-exfoliated $\mathrm{MoS}_{2}$ flake with a lateral size, $L$, of $\sim 5 \mu \mathrm{m}$ and a thickness, $d$, of $\sim 8 \mathrm{~nm}$ (corresponding critical 


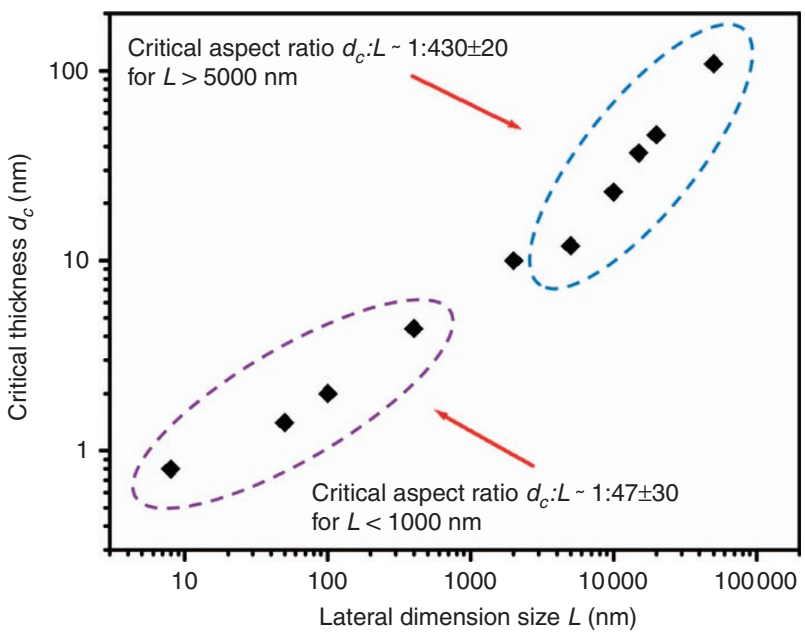

Figure 5 Critical thickness $\left(d_{c}\right)$ values plotted as a function of the corresponding characteristic lateral dimension sizes $(L)$.

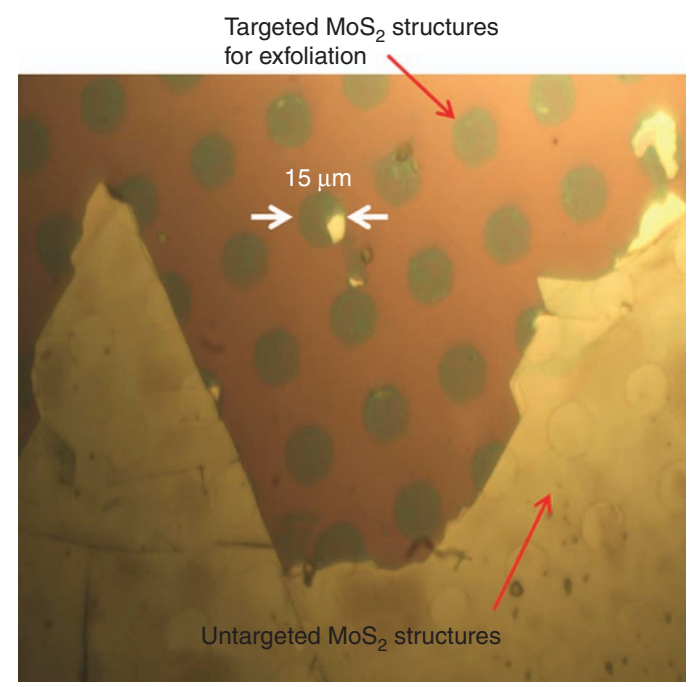

Figure 6 Optical micrograph of as-exfoliated $15 \mu \mathrm{m}$-sized, $20 \mathrm{~nm}$ thick $\mathrm{MoS}_{2}$ mesas and untargeted $\mathrm{MoS}_{2}$ layers, which are exfoliated due to the poor exfoliation selectivity between the pre-patterned (or targeted) and unpatterned (or untargeted) $\mathrm{MoS}_{2}$ layers. This poor selectivity is usually observed when the aspect ratio of prepatterned to-be-exfoliated $\mathrm{MoS}_{2}$ structures is close to or smaller than the critical value.

thickness, $d_{c}$ of $\sim 10 \mathrm{~nm}$ ) and the other flake with a $L$ of $\sim 5 \mu \mathrm{m}$ and a $d$ of $\sim 18 \mathrm{~nm}\left(d_{\mathrm{c}}\right.$ is still $\left.\sim 10 \mathrm{~nm}\right)$, respectively. The $\mathrm{MoS}_{2}$ flake with $d \sim d_{c}$ exhibits a quasi-periodic Moiré pattern on its cleaved surface, which indicates the existence of exfoliation-induced interlayer twist features in the $\mathrm{MoS}_{2}$ layers close to the cleaved surface. The cleaved surface of the $\mathrm{MoS}_{2}$ flake with $d>d_{c}$ does not exhibit any Moiré patterns but features a high-quality crystal lattice, indicating no exfoliation-induced interlayer deformation or twisting in the exfoliated layers.

The interlayer twisting angle $(\theta)$ and the resulting Moiré pattern period $(P)$ in $\mathrm{MoS}_{2}$ layers can be evaluated using $P=a / \theta$ in which $a$ is the in-plane lattice constant (for $\mathrm{MoS}_{2}, a=0.318 \mathrm{~nm}$ ). The Moiré pattern periods were observed in the $\mathrm{MoS}_{2}$ flake with the $d \sim d_{\mathrm{c}}$ range from 3 to $6 \mathrm{~nm}$, from which the interlayer twisting angles were estimated to be $3-6^{\circ}$. As implied by several previous theoretical works, the Moiré patterns in few-layer TMDC structures could spatially modulate the interlayer coupling of electronic band states that originated from different layers, resulting in spatial trapping of charged carriers and spatial variation of electrostatic potential $^{16-18}$. Such a Moiré pattern effect, if introduced into a field-effect transistor structure, is expected to result in charge memory states. To support this speculation, we further investigated and compared the charge-trapping characteristics of the FETs made from the $\mathrm{MoS}_{2}$ flakes in the cases of $d \sim d_{\mathrm{c}}$ and $d>d_{\mathrm{c}}$. Figure 7c schematically illustrates the back-gated $\mathrm{MoS}_{2}$ FET structure under study. For such an FET, the $\mathrm{MoS}_{2}$ channel length is $\sim 4 \mu \mathrm{m}$, and the channel width is $\sim 5 \mu \mathrm{m}$. A pair of Ti $(5 \mathrm{~nm}) / \mathrm{Au}$ $(50 \mathrm{~nm})$ electrodes were fabricated using photolithography followed by metallization to serve as source-drain (D-S) contacts. The $\mathrm{p}^{+}-$Si substrate serves as the back gate (G) for modulating the $\mathrm{MoS}_{2}$ channel. The thermally grown $300 \mathrm{~nm}$-thick $\mathrm{SiO}_{2}$ layer on top of the Si substrate serves as the gate dielectric. The FETs were characterized using a LakeShore probe station (Lake Shore Cryotronics Inc., Westerville, OH) connected to a HP4145 semiconductor parameter analyzer. Figures $7 d$ and e display the optical micrographs of two representative $\mathrm{MoS}_{2}$ FETs in the cases of $d \sim d_{\mathrm{c}}$ and $d>d_{\mathrm{c}}$, respectively (that is, the FETs made from the $\mathrm{MoS}_{2}$ flakes very similar to the ones shown in Figures 7a and b). Figures $7 f$ and $g$ show the drain-source current $\left(I_{\text {DS }}\right)$ - gate voltage $\left(V_{\mathrm{G}}\right)$ curves (or transfer characteristic curves) of these two devices. The transfer curves in Figure $7 \mathrm{~g}$ exhibit a couple of small kinks. These kinks should be attributed to the interfacial states at the $\mathrm{MoS}_{2} / \mathrm{SiO}_{2}$ interfaces. The charging or discharging events associated with these states usually result in small kink features on transfer characteristic curves. However, such charge-trapping states have very short retention times and do not prominently affect the charge retention characteristics of interlayer deformation-induced charge-trapping states in $\mathrm{MoS}_{2}$ layers. The On/Off current ratio of the FET with $d \sim d_{\mathrm{c}}\left(I_{\mathrm{ON}} / l_{\mathrm{OFF}} \sim 10^{4}\right)$ is slightly larger than that of the one with $d>d_{\mathrm{c}}\left(I_{\mathrm{ON}} / I_{\mathrm{OFF}} \sim 10^{3}\right)$. This is because the thinner $\mathrm{MoS}_{2}$ FET channel usually results in a higher effectiveness of the gate potential for modulating the channel conductance. However, for both FETs, their On/Off ratios are high enough to enable a dynamic range of the channel conductance (or $I_{D S}$ measured under a given set of $V_{D S}$ and $V_{G}$ biases) for observing the $V_{\mathrm{G}}$-mediated charge-trapping processes in the FETs. Specifically, a queue of $-50 \mathrm{~V}, 100-\mathrm{ms} V_{\mathrm{G}}$ pulses with pulseto-pulse spacing of $30 \mathrm{~s}$ were sequentially applied to the FET under study. During the intervals between $V_{G}$ pulses, the timedependent $I_{\mathrm{DS}}$ values (that is, $I_{\mathrm{DS}}-t$ curves) were measured under fixed $V_{D S}$ and $V_{G}$ (typically $V_{G}=0$ and $V_{D S}=0.05 \mathrm{~V}$ ) to show the $V_{\mathrm{G}}$-mediated charge-trapping processes in the FET. Figures $7 \mathrm{~h}$ and i display the $I_{\mathrm{DS}}-t$ curves measured from the $\mathrm{MoS}_{2}$ FETs in the cases of $d \sim d_{c}$ and $d>d_{c}$, respectively. We observed that the FET with $d \sim d_{c}$ exhibits a cascading increase of $I_{D S}$ in response to the time-sequenced $V_{G}$ pulses, whereas the FET with $d>d_{c}$ does not show a significant change in the $I_{\mathrm{DS}}$ level, and the slight fluctuation in $I_{D S}$ is not relevant for the applied $V_{G}$ pulses. This characterization result shows that the FET with $d \sim d_{c}$ has $V_{\mathrm{G}}$-tunable charge-trapping states that do not exist in the FET with $d>d_{\mathrm{c}}$. In one of our recent works, we found that few-layer $W \mathrm{We}_{2}$ FETs, once they bear interlayer deformation nanostructures, exhibit multiple long-lasting charge-trapping states ${ }^{9}$. Therefore, the $V_{\mathrm{G}}$-tunable charge-trapping states observed in the present work can also be reasonably attributed to the interlayer twisting features observed in the $\mathrm{MoS}_{2}$ channel with $d \sim d_{c}$. This FET result is consistent with the TEM result mentioned above and further indicates that the NASE+TP-produced $\mathrm{MoS}_{2}$ structures with feature thickness close to $d_{c}$ likely bear interlayer twisting or other deformation features, which can result in $V_{\mathrm{G}}$-tunable chargetrapping states in the FETs made from such $\mathrm{MoS}_{2}$ structures. Such charge-trapping states could be further studied for making multibit or quasi-analog-tunable memory devices for emerging neuromorphic and analog computing device applications. In addition, our $\mathrm{MoS}_{2}$ FETs with lengths/widths in the range of 

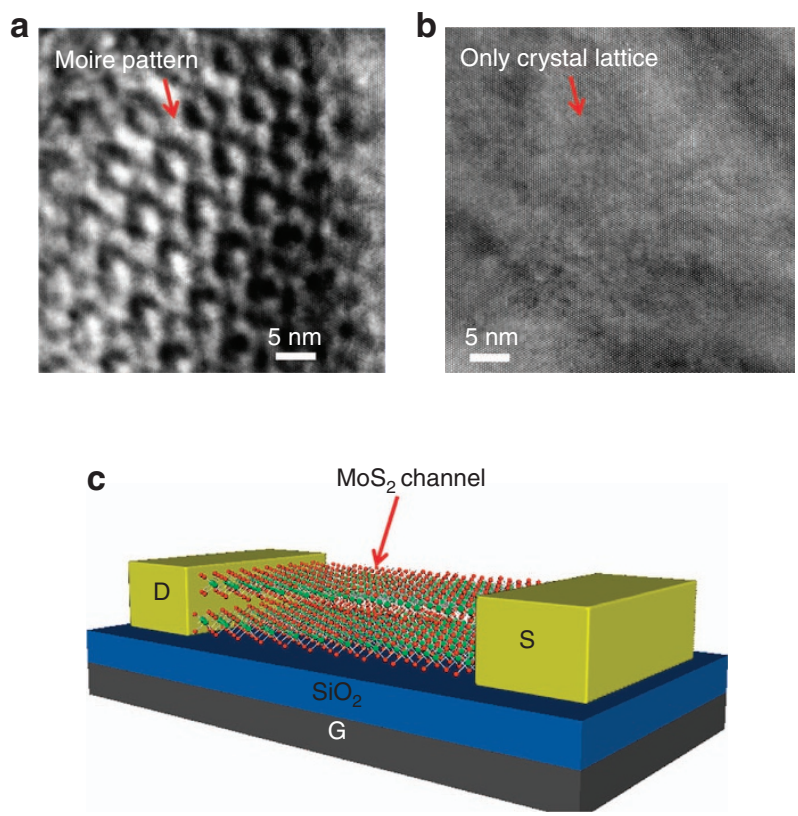

d

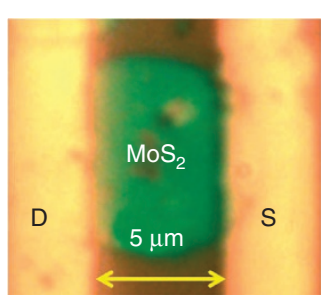

f

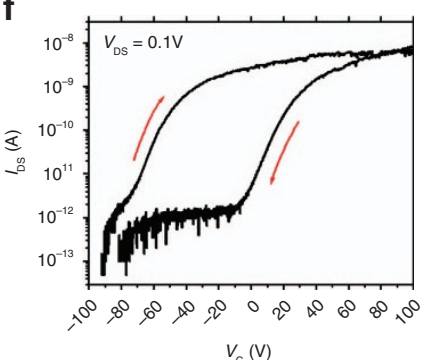

h

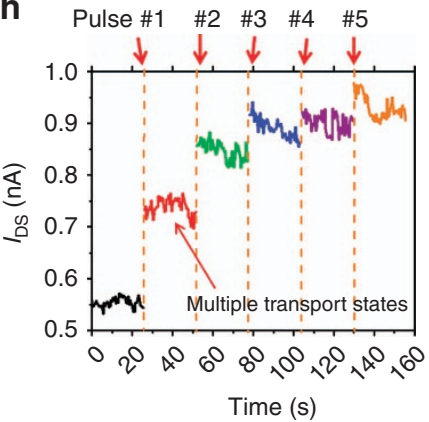

e

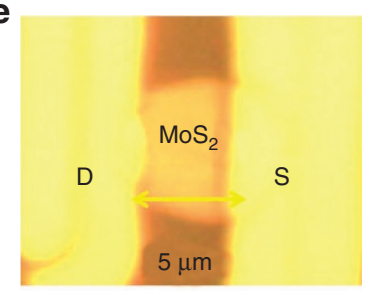

g
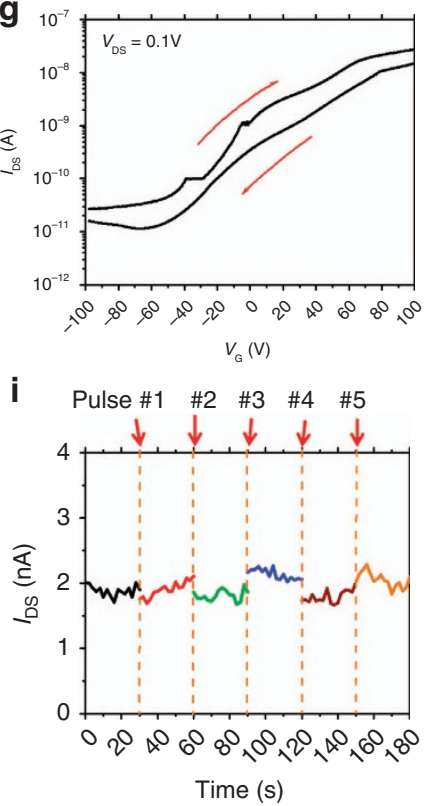

Figure 7 Charge memory characteristics of the FETs made from $\mathrm{MoS}_{2}$ flakes: (a) and (b) show the HRTEM images of the cleaved surfaces of one $\mathrm{MoS}_{2}$ flake of critical thickness (or critical aspect ratio) and the other flake, which is thicker than the critical thickness, respectively; (c) schematic illustration of a back-gated $\mathrm{MoS}_{2}$ FET; (d and e) show the optical micrographs of the FETs made from the MoS ${ }_{2}$ flakes shown in (a and $\mathbf{b}$ ), respectively; ( $\mathbf{f}$ and $\mathbf{g}$ ) display $I_{\mathrm{DS}}-V_{\mathrm{G}}$ curves measured from these two FETs, respectively; (h and i) display the $I_{\mathrm{DS}}-t$ curves measured from the FETs made from the $\mathrm{MoS}_{2}$ flakes shown in (a and $\mathbf{b}$ ), respectively, which are modulated by a set of periodic $-50 \mathrm{~V}, 100 \mathrm{~ms} V_{\mathrm{G}}$ pulses.

2-20 $\mu \mathrm{m}$, if their aspect ratios are close to the corresponding critical values, exhibit very similar charge-trapping characteristics. However, it is still not clear whether or not the $\mathrm{MoS}_{2}$ FETs with nanoscale lengths or widths have similar charge-trapping states due to interlayer deformation. To fully understand this question, we need methods for making nanoscale $\mathrm{MoS}_{2}$ FETs without populating the charging states associated with other sources. In particular, in our current fabrication method, the plasma etching processes always introduce additional charge-trapping states at the feature edges, which dominate the charging characteristics of nanoscale $\mathrm{MoS}_{2}$ FETs (note that such charging states at the feature edges are not dominant in microscale $\mathrm{MoS}_{2}$ FETs.) In future work, we will seek to rule out the effects of such charging states at the feature edges of nanoscale $\mathrm{MoS}_{2}$ FETs and therefore identify the interlayer deformation-mediated charge-trapping characteristics for these types of nanoscale devices.

\section{CONCLUSIONS}

In summary, we studied the critical limit of the feature dimensions of $\mathrm{MoS}_{2}$ nano- and microstructures produced via NASE+TP processes. In particular, we explored the processing condition for producing ultrathin monolayer and few-layer $\mathrm{MoS}_{2}$ structures. Our work shows that the quality and yield of NASE+TP-produced $\mathrm{MoS}_{2}$ nano- and microstructures are dependent on the aspect ratio of pre-patterned to-be-exfoliated $\mathrm{MoS}_{2}$ structures on a bulk stamp. For the pre-patterned $\mathrm{MoS}_{2}$ structures with a given lateral feature size, the vertical thickness (or aspect ratio) of these structures has a critical limit; below this critical limit, the asexfoliated $\mathrm{MoS}_{2}$ nano- and microstructures are easily damaged by the NASE processes. Our experiments specifically indicate that for $\mathrm{MoS}_{2}$ structures with lateral feature sizes of $>5 \mu \mathrm{m}$, the critical aspect ratio is estimated to be approximately $1: 430$, whereas for $\mathrm{MoS}_{2}$ nanostructures with lateral sizes of $<1 \mu \mathrm{m}$, the critical aspect ratio is approximately $1: 47$. This indicates that to generate ultrathin monolayer or few-layer $\mathrm{MoS}_{2}$ structures that are free of mechanical damage, the lateral dimensions of the protrusive $\mathrm{MoS}_{2}$ structures on the stamp need to be pre-patterned in the sub-100$\mathrm{nm}$ regime. Furthermore, we found that the as-exfoliated $\mathrm{MoS}_{2}$ structures with thicknesses or aspect ratios very close to the corresponding critical values bear interlayer twisting features that result in top-view Moiré patterns on the cleaved surfaces. The FETs made from these $\mathrm{MoS}_{2}$ layers exhibit multiple $V_{\mathrm{G}}$-tunable charge memory states. This work advances the understanding of the limitation and application scope of nanoimprint and nanoprint processes for manufacturing $\mathrm{MoS}_{2}$ and other TMDC device structures. The identified charge memory states in the $\mathrm{MoS}_{2}$ FETs with thicknesses close to the critical values could be further studied and exploited to make analog computing and multi-bit memory devices.

\section{ACKNOWLEDGEMENTS}

This work was supported by NSF grant \# CMMI-1636132. We thank Dr Kai Sun at the Michigan Center for Materials Characterization (MC2) and Ph.D student, Da Li for providing the support for the SEM imaging and EDS characterization. 


\section{COMPETING INTERESTS}

The authors declare no conflict of interest.

\section{REFERENCES}

1 Radisavljevic B, Radenovic A, Brivio J et al. Single-layer $\mathrm{MoS}_{2}$ transistors. Nature Nanotechnology 2011; 6: 147-150.

2 Mak KF, Lee C, Hone J et al. Atomically thin $\mathrm{MoS}_{2}$ : A new direct-gap semiconductor. Physical Review Letters 2010; 105: 136805/136801-136805/136815.

3 Korn T, Heydrich S, Hirmer M et al. Low-temperature photocarrier dynamics in monolayer MoS 2 . Applied Physics Letters 2011; 99: 102109/102101-102109/102103.

4 Chhowalla M, Shin HS, Eda G et al. The chemistry of two-dimensional layered transition metal dichalcogenide nanosheet. Nature Chemistry 2013; 5: 263-275.

5 Wang $\mathrm{H}, \mathrm{Yu}$ L, Lee Y-H et al. Integrated circuits based on bilayer $\mathrm{MoS}_{2}$ transistors. Nano Letters 2012; 12: 4674-4680.

$6 \mathrm{Nam} \mathrm{H}$, Oh BR, Chen PY et al. Multiple $\mathrm{MoS}_{2}$ transistors for sensing molecule interaction kinetics. Scientific Reports 2015; 5: 10546/10541-10546/10513.

$7 \mathrm{Nam} \mathrm{H}$, Oh BR, Chen P et al. Two different device physics principles for operating $\mathrm{MoS}_{2}$ transistor biosensors with femtomolar-level detection limits. Applied Physics Letters 2015; 107: 012105/012101-012105/012105.

$8 \mathrm{Nam} \mathrm{H}$, Oh BR, Chen MK et al. Fabrication and comparison of $\mathrm{MoS}_{2}$ and $\mathrm{WSe}_{2}$ field-effect transistor biosensors. Journal of Vacuum Science and Technology B 2015; 33: 06FG01/01-06FG01/07.

9 Chen $\mathrm{M}$, Wang $\mathrm{Y}$, Shepherd $\mathrm{N}$ et al. Abnormal multiple charge memory states in exfoliated few-layer WSe 2 transistors. ACS Nano 2017; 11: 1091-1102.

10 Chen $M$, Nam H, Rokni $\mathrm{H}$ et al. Nanoimprint-assisted shear exfoliation (NASE) for producing multilayer $\mathrm{MoS}_{2}$ structures as field-effect transistor channel arrays. ACS Nano 2015; 9: 8773-8785.

11 Li D, Wi S, Chen M et al. Nanoimprint-assisted shear exfoliation plus transfer printing for producing transition metal dichalcogenide heterostructures. Journal of Vacuum Science and Technology B 2016; 34: 06KA01.
$12 \mathrm{Nam} \mathrm{H}$, Wi S, Rokni $\mathrm{H}$ et al. $\mathrm{MoS}_{2}$ transistors fabricated via plasma-assisted nanoprinting of few-layer $\mathrm{MoS}_{2}$ flakes into large-area arrays. ACS Nano 2013; 7: 5870-5881.

13 Liang XG, Wi S. Transport characteristics of multichannel transistors made from densely aligned sub-10 nm half-pitch graphene nanoribbons. ACS Nano 2012; 6: 9700-9710.

14 Park SM, Liang XG, Harteneck BD et al. Sub-10 nm nanofabrication via nanoimprint directed self-assembly of block copolymers. ACS Nano 2011; 5: 8523-8531.

15 Liang XG, Jung YS, Wu SW et al. Formation of bandgap and subbands in graphene nanomeshes with Sub-10 nm ribbon width fabricated via nanoimprint lithography. Nano Letters 2010; 10: 2454-2460.

16 Kang J, Li J, Li SS, Xia JB, Wang LW. Electronic structural moire pattern effects on $\mathrm{MoS}_{2} / \mathrm{MoSe}_{2} 2 \mathrm{D}$ heterostructures. Nano Letters 2013; 13: 5485-5490.

17 Decker R, Wang Y, Brar VW et al. Local electronic properties of graphene on a BN substrate via scanning tunneling microscopy. Nano Letters 2011; 11: 2291-2295.

18 Woods CR, Britnell L, Eckmann A et al. Commensurate-incommensurate transition in graphene on hexagonal boron nitride. Nature Physics 2014; 10: $451-456$.

cc) (i)

This work is licensed under a Creative Commons Attribution 4.0 International License. The images or other third party material in this article are included in the article's Creative Commons license, unless indicated otherwise in the credit line; if the material is not included under the Creative Commons license, users will need to obtain permission from the license holder to reproduce the material. To view a copy of this license, visit http://creativecommons.org/licenses/ by/4.0/

(c) The Author(s) 2017 